Revue d'histoire de l'enfance " irrégulière »

Le Temps de l'histoire

$17 \mid 2015$

Naissance et mutation de la justice des mineurs

Juvenile courts américaines et tribunaux pour enfants français : les variations d'un modèle à travers la comparaison Paris/Boston (début XXe siècle-années 1950)

American juvenile courts and French tribunaux pour enfants: model's variations (a comparison between Paris and Boston, early 20th century-1950s)

Guillaume Périssol

\title{
OpenEdition
}

Journals

Édition électronique

URL : http://journals.openedition.org/rhei/3819

DOI : 10.4000/rhei.3819

ISSN : $1777-540 \mathrm{X}$

Éditeur

Presses universitaires de Rennes

Édition imprimée

Date de publication : 30 octobre 2015

Pagination : 79-99

ISBN : 978-2-7535-4215-0

ISSN : 1287-2431

Référence électronique

Guillaume Périssol, « Juvenile courts américaines et tribunaux pour enfants français : les variations d'un modèle à travers la comparaison Paris/Boston (début xxe siècle-années 1950) ", Revue d'histoire de l'enfance « irrégulière » [En ligne], 17 | 2015, mis en ligne le 30 octobre 2017, consulté le 10 décembre 2020. URL : http://journals.openedition.org/rhei/3819; DOI : https://doi.org/10.4000/rhei.3819 


\section{Juvenile courts américaines et tribunaux pour enfants français: les variations d'un modèle à travers la comparaison Paris/Boston (début Xxe siècle - années 1950)}

L'exposé des motifs de la loi du 22 juillet 1912, créant les tribunaux pour enfants en France, cite en " exemple » les États-Unis comme «le type le plus achevé de cette méthode plus humaine ». C'est en effet dans l'Amérique de la Progressive Era que naît, en 1899, à Chicago, le premier tribunal des enfants. Le modèle de la juvenile court connaîtra rapidement un succès mondial, avec des déclinaisons nationales et, dans un État fédéral comme les États-Unis, des variations locales, d'un État à l'autre. En comparant les tribunaux pionniers et dynamiques de deux capitales, de la France et du Massachusetts, l'objectif est de repérer quelques éléments structuraux de cette nouvelle forme de justice, « plus humaine ", pour la période allant du début du $x x^{e}$ siècle aux années 1950. Afin de dresser un panorama et d'expliquer l'impact et le retentissement du modèle de la juvenile court, seront successivement analysés, à partir d'une recherche en cours, les cadres juridiques, les populations visées et les pratiques de jugement à Paris et à Boston.

The explanatory statement for the July 22, 1912 Law establishing juvenile courts in France held up the United States as an "example" of the "most advanced type of this more humane method." The first juvenile court was created in 1899, in Chicago, United States, during the Progressive Era. The model of the juvenile court rapidly became a worldwide success with national variations, and local and State variations in the United States, a federal country. The article will compare the pioneering and dynamic courts of two capital cities - of France and of the state of Massachusetts. The aim is to identify certain structural features of this novel form of "more human" justice, from the early 20th century to the 1950s. In order to provide an overview of the model of the juvenile court and explain its impact and repercussions, we will analyze (on the basis of ongoing research) legal frameworks, populations targeted and judges' decisions in Paris and Boston.
Guillaume PérISSOL Doctorant en histoire à l'université ParisSorbonne. Attaché temporaire d'enseignement et de recherche au département des sciences de l'éducation de l'université Paris 8 - Vincennes - SaintDenis. Chercheur invité (lauréat Arthur Sachs) en 2012 au département d'histoire de l'université Harvard.

Une version anglaise de cet article a fait l'objet d'une publication sous la référence: PERISSOL

Guillaume, "The

Quality of Mercy is not

Strain'd": Ideological and Repressive modes of juvenile justice -

A Comparison between Paris and Boston in the Mid-Twentieh Century", Journal of social history, vol. 48, n०2, Winter 2014, p. 289-312. 
1. Remarque: sauf précision, le mot « américain » sera employé relativement aux États-Unis d'Amérique.

2. JULHIET Édouard, «LeS tribunaux pour enfants aux États-Unis ", Les tribunaux spéciaux pour enfants, Paris, Administration de la Revue l'Enfant, Collection de la Revue I'Enfant, 1906,

p. 10. Disponible sur: [www. archive.org/download/ lestribunauxspc00gastgoog/ lestribunauxspc00gastgoog pdf] (consulté le 29 août 2013)

3. Ibid., p. 20-23. Édouard Julhiet entend la prison au sens fort du terme (les maisons de réforme, de correction, etc., restant des solutions possibles, même si la "sentence " rendue "le plus souvent », et « qui constitue la principale raison d'être des tribunaux pour enfants », est la mise en liberté surveillée).

4. Ibid., p. 10

5. En 1923 paraissent les Juvenile-Court Standards (Normes des tribunaux pour enfants), réimprimés et distribués par le Children's Bureau, organisme fédéral, jusqu'en 1954

6. Au recensement de 1900, 34,6\% des habitants de Chicago sont nés à l'étranger (foreign born), la population noire étant encore faible (moins de $5 \%$ des habitants)
Mots-clefs: justice des mineurs, tribunaux pour enfants, délinquance juvénile, histoire comparée, France, États-Unis, $x x^{e}$ siècle Keywords: juvenile justice, juvenile courts, juvenile delinquency, comparative history, France, United States, 20th century

T 'exposé des motifs de la loi du 22 juillet 1912, _qui crée les tribunaux pour enfants (TE) en France, cite en "exemple » les États-Unis comme "le type le plus achevé de cette méthode plus humaine ». Six ans avant, Édouard Julhiet, dans sa fameuse conférence au Musée social où il présentait le fonctionnement encore mal connu des TE américains ${ }^{1}$, définissait le "tribunal-type ${ }^{2}$ " par trois éléments de base: " la spécialisation du tribunal ", " la suppression de la prison pour les enfants » (de la " prison commune », en tous cas $^{3}$ ) et " la mise en liberté surveillée ", qui traduit le terme anglais de probation. Mais il rappelait que " les tribunaux pour enfants aux États-Unis sont très différents les uns des autres ${ }^{4}$ ». Le fédéralisme laisse, en effet, à chaque État l'essentiel des compétences en matière de justice des mineurs et, si des normes peuvent être posées au niveau fédéral, comme ce sera le cas en $1923^{5}$, elles n'ont pas force de contrainte.

La première réalisation a lieu en 1899 à Chicago. Dans cette ville en ébullition, passée en 50 ans d'environ 30000 à près de 1700000 habitants (au deuxième rang national après New York), comptant plus d'un tiers d'immigrés ${ }^{6}$, l'exploitation capitaliste produit fortunes rapides et misère, tensions et violences. Des philanthropes proposent des solutions pacifiques et innovantes aux risques 
de chaos social: la juvenile court (tribunal pour enfants) est l'une d'elles. Au Congrès pénitentiaire international de Washington, en 1910, le TE du Cook County de Chicago, et les autres TE américains nés depuis, sont salués comme la "révélation d'un progrès et d'une vérité dont tous les États du globe doivent faire leur profit ${ }^{7}$ ". Le succès va croissant, au niveau national et international. En 1925, tous les États américains, sauf deux, le Maine et le Wyoming, ont adopté des lois relatives à la justice des mineurs, et des juvenile courts fonctionnent dans toutes les villes d'au moins 100000 habitants ${ }^{8}$.

Le Massachusetts est habituellement pionnier dans le domaine judiciaire; c'est là qu'au $\mathrm{XIX}^{\mathrm{e}}$ siècle est inventée la probation dans sa forme moderne et que se crée, à Westborough, en 1846, la première " école de réforme " publique, partiellement inspirée de la colonie de Mettray. Cet État institue donc rapidement la Boston Juvenile Court (BJC), en $1906^{9}$. Ce tribunal spécialisé a une juridiction limitée aux principaux quartiers de Boston, du centre-ville essentiellement; ailleurs, dans le Massachusetts, la justice des mineurs est rendue par les tribunaux de districts lors de sessions spécifiques. Cela montre bien les différences de traitement qui peuvent exister au sein d'un même État et pas seulement d'un État à l'autre, la délinquance juvénile étant d'abord pensée comme un problème urbain. De même, en France, avant l'ordonnance du 2 février 1945 , seules certaines villes se dotent de TE. Ainsi, le TE de la Seine se met en place en 1914 pour les
7. Discours d'H.-C Dresselhuys (Pays-Bas), à Chicago, lors du voyage d'études aux États-Unis organisé à l'occasion du Congrès pénitentiaire de Washington en 1910, dans: Procès-verbaux des séances et voyages d'études, etc., vol. 1 des Actes du Congrès pénitentiaire international de Washington, octobre 1910, Groningen, Bureau de la Commission pénitentiaire internationale, 1913, p. 570. Disponible sur: http:// data.decalog.net/enap1/ liens/congres/CONGRES_ PENIT_1910_VOL1_0002. pdf (consulté le 26 février 2013).

8. LENROOT Katharine F. and LUNDBERG Emma O., Juvenile Courts at Work: a Study of the Organization and Methods of Ten Courts,
Washington, Government Printing Office, 1925, graphique p. 2. Disponible sur: [http://www.archive. org/details/juvenilecourtsat00unit] (consulté le 11 août 2013).

9. Le troisième TE américain selon KLAYMAn Richard, «The Boston Juvenile Court and the Progressive Challenge of Child-Saving, 19061986 », Historical Journal of Massachusetts, vol. 16, $\mathrm{n}^{\circ} 2$, Summer 1988, p. 205 Une erreur de l'auteur, puisqu'existent déjà en 1906 les TE de Chicago, Philadelphie, Denver, etc. Édouard Julhiet note que « cinq ans et demi après la création du premier tribunal d'enfants ", soit en 1905, 24 États américains ont déjà " adopté ce nouveau rouage judiciaire » (JULHIET, « LeS tribunaux pour enfants... ", p. 8). 
10. Par exemple le $n^{\circ} 5$, de 2003, de la Revue d'histoire de l'enfance «irrégulière » (RHEI): Pratiques éducatives et systèmes judiciaires. Signalons aussi : DUPONT-BOUChat Marie-Sylvie et Pierre Éric (dir.), Enfance et justice au XIX siècle siècle: essais d'histoire comparée de la protection de l'enfance, 1820-1914, France, Belgique, Pays-Bas, Canada, Paris, PUF, Droit et justice, 2001, $443 \mathrm{p}$.

11. Niget David, La naissance du tribunal pour enfants: une comparaison France-Québec (19121945), Rennes, Presses universitaires de Rennes, Histoire, 2009, 417p.

12. On trouvera une bonne mise au point historiographique autour et au-delà du livre de Platt dans sa réédition anniversaire de 2009:

Chávez-García Miroslava, « In Retrospect: Anthony M. Platt's The Child Savers: The Invention of Delinquency ", PLATT Anthony M. The Child Savers: The Invention of Delinquency, expanded $40^{\text {th }}$ anniversary edition, New Brunswick, New Jersey, Rutgers University Press, 2009, p. XI-XXXVI.

13. ChazAl Jean, « Vers un néohumanisme judiciaire ",

chap. II des Études de criminologie juvénile, Paris, PUF, 1952, p. 6 sq. populations de Paris et de la proche banlieue, parvenant à fonctionner en juridiction relativement spécialisée et autonome.

Du point de vue historiographique, l'histoire comparative des TE est peu développée et presque rien n'existe sur la comparaison France/États-Unis. Côté français, en dehors de quelques études ou numéros spéciaux de revues ${ }^{10}$, il faut citer, pour le $\mathrm{xx}^{\mathrm{e}}$ siècle, le travail pionnier de David Niget ${ }^{11}$ qui porte sur la France et le voisin canadien, mais aborde la problématique américaine, intégrant une partie de sa littérature. Côté américain, la production est relativement importante, mais centrée sur les États-Unis de la Progressive Era (ère progressiste), c'est-à-dire sur la période de création et de développement initial des juvenile courts. L'ouvrage d'Anthony M. Platt de 1969 sur les child savers (sauveurs d'enfants) a suscité une littérature abondante autour des thèses de contrôle social qu'il développait ${ }^{12}$. Les TE ont en fait beaucoup servi aux États-Unis pour comprendre et critiquer le soi-disant progressisme du tournant des $\mathrm{XIX}^{\mathrm{e}}$ et $\mathrm{Xx}^{\mathrm{e}}$ siècles. La problématique est restée très américaine et les textes normatifs ont longtemps été favorisés par rapport aux archives (parfois pour des raisons liées à leur conservation ou à leur accès), ce qui fait qu’on ne connaît pas très bien les pratiques d'un tribunal à l'autre, les éventuelles résistances des acteurs, etc.

Le comparatisme aide pourtant à répondre à ces questions : que cachent le progressisme des juvenile courts, la «méthode plus humaine » reprise par la loi de 1912, le «néohumanisme judiciaire ${ }^{13}$ " vanté par le juge français Chazal après l'ordonnance de 1945? Que signifie le succès extrêmement rapide des TE en Occident et dans le monde? À quelles nécessités répond-il?

En comparant les tribunaux pionniers et dynamiques de deux capitales, de la France et du Massachusetts, l'objectif est de repérer quelques éléments structuraux de cette nouvelle forme de justice pour la période allant du début du Xx siècle aux années 1950, puisqu’à partir des années 1960, aux États-Unis, l'histoire des TE bascule: la critique radicale ou libérale (au sens américain) va malencontreusement rencontrer la critique conservatrice. Une série d'arrêts progressistes, et protecteurs des droits de la défense, de la Cour suprême des États-Unis, dont les célèbres Kent et Gault de 1966 et 1967, engendre des effets pervers dans les années 1980 et 1990, en pleine vague d' "insécurité " abondamment médiatisée, favorisant des réformes législatives qui aboutiront à une très forte remise en cause, parfois une quasi-destruction, du modèle dans 
le pays qui l'a créé. Les États-Unis renouent alors avec la répression et l'enfermement des mineurs à un niveau qu'aucun autre pays occidental n'a plus connu, ne regagnant du bon sens que récemment - par exemple, avec l'arrêt Simmons de la Cour suprême des États-Unis, en 2005, contre l'application de la peine de mort aux moins de 18 ans.

Afin de dresser un panorama pour cette première moitié du $\mathrm{xx}^{\mathrm{e}}$ siècle et d'expliquer l'impact et le retentissement du modèle de la juvenile court, seront successivement analysés à partir d'une recherche en cours: les cadres juridiques, les populations visées et les pratiques de jugement.

\section{LES LOIS ET TRADITIONS JURIDIQUES}

Dans le Massachussets, une loi d'État crée en 1906 la BJC avec des juges spécialisés, appliquant la doctrine du parens patriae ${ }^{14}$ : sauf cas graves, les enfants seront traités comme ils seraient traités par des parents. La solution pénale est normalement rejetée, comme le révèle le changement de vocabulaire (le mineur n'est plus guilty ou not guilty, coupable ou non-coupable, il est ou non delinquent) ou la conception immédiatement très large des populations de mineurs pris en charge par la juvenile court, agissant en parent supplétif de l'enfant délinquant, négligé, etc. ${ }^{15}$, brouillant ainsi les frontières entre enfance coupable et enfance en danger. En France, la justice des mineurs se construit en deux principales étapes législatives: la loi de 1912 et surtout l'ordonnance de 1945, qui constitue le véritable acte de naissance des TE avec l'instauration de juges spécialisés sur l'ensemble du territoire national.
14. Dans LENROOT

Katharine F. and LUNDBERG Emma O., Juvenile Courts... p. 126 sq., sont distinguées les procédures " quasipénales ", comme celles de la BJC, et les procédures de " chancery courts " (cours de chancellerie) auxquelles les TE font le plus souvent référence; la pratique est en fait assez proche et la doctrine du parens patriae généralisée.

15. Dans Van De Kerchove Michel, Le Droit sans peines: aspects de la dépénalisation en Belgique et aux États-Unis, Bruxelles, Facultés universitaires Saint-Louis, Publications des Facultés universitaires Saint-Louis, 1987, p. 136 et p. 138 , l'auteur note que le titre officiel de la loi de 1899, créant le premier TE, est An Act to Regulate the Treatment and Control of Dependent, Neglected and Delinquent Children (Loi réglementant le traitement et le contrôle des enfants délinquants, négligés et délaissés); citant Frederick B. Sussman, il rappelle également la variabilité, d'un État à l'autre, de la définition juridique de la délinquance juvénile. Dans le Massachusetts, les dependent children (enfants délaissés, pris en charge par l'État) ne sont pas gérés par le TE, sauf s'ils tombent dans la catégorie de l'enfant négligé ainsi définie: enfant de moins de 16 ans qui, " en raison de son statut d'orphelin ou en raison de la négligence, délinquance [crime], cruauté, aliénation mentale, alcoolisme ou autre vice de ses parents, grandit sans éducation ou sans contrôle bénéfique [salutary] ou sans soins corporels appropriés ou dans des circonstances l'exposant à une vie dissolue ou de paresse, ou dépend de la charité publique " (Massachusetts, Annotated Laws of Massachusetts, 1949, chap. 119, §42). 
16. GLUECK Sheldon and GLUECK Eleanor T., One Thousand Juvenile Delinquents: Their Treatment by Court and Clinic, Cambridge, Harvard University Press, 1934, XIX-341p

17. Renommée plus tard: Judge Baker Guidance Clinic.

18. Citons, entre autres expériences comparables, la Clinique d'aide à l'enfance, instituée par une loi de 1945, qui est directement attachée à la Cour des jeunes délinquants de Montréal, au Canada.

19. Foucault Michel, Surveiller et punir: naissance de la prison, Paris, Gallimard, Bibliothèque des Histoires, 1975, p. 311.

20. SCHNeIDER Eric C., In the Web of Class: Delinquents and Reformers in Boston, 1810s-1930s, New York, New York University Press, The American Social Experience, 1992, p. 172.
Les origines et les mythes des origines se situent donc à plusieurs dizaines d'années de distance en France et aux États-Unis. Outre-Atlantique, le modèle du TE est sérieusement attaqué dès l'entre-deux-guerres, dans le contexte de la Grande Dépression et de la grave crise sociale qu'elle provoque. En 1934, au moment où deux criminologues d'Harvard, les Glueck, mettent en cause l'efficacité des TE à partir d'une recherche ${ }^{16}$ menée sur 1000 garçons passés par les circuits de la BJC, calculant un taux de récidive de près de $90 \%$, en France, Prévert écrit un poème, La chasse à l'enfant, en soutien à la campagne médiatique en cours contre les " bagnes d'enfants ", c'est-à-dire contre le modèle disciplinaire du XIX ${ }^{\mathrm{e}}$ siècle.

En 1945, le TE est encore une innovation en France; aux États-Unis, l'optimisme de départ s'est émoussé et l'attrait de la nouveauté n'est plus là, expliquant peut-être la moindre présence des Américains sur la scène internationale après la seconde guerre mondiale.

Le TE fonctionne au sein de ce que les Américains nomment le juvenile justice system (système de justice pour mineurs), car il nécessite des services et convoque des savoirs pour la rééducation du mineur. L’entre-deux-guerres voit le développement rapide du système, de manière relativement concomitante à Boston et à Paris. Du point de vue des sciences du psychisme par exemple, un mouvement de création de clinics s'enclenche: dès 1909 s'ouvre le Juvenile Psychopathic Institute à Chicago, première institution du genre dans le pays, dirigé par le docteur William Healy, qui, en 1917, est appelé à Boston pour prendre la tête, avec le docteur Augusta Bronner, sa future femme, de la Judge Baker Foundation ${ }^{17}$; en France, en 1925 est fondée la Clinique de neuropsychiatrie infantile, dirigée par le docteur Georges Heuyer, au sein du Patronage Rollet. Le mouvement est mondial ${ }^{18}$, répondant à la logique même de la justice exploratoire (ou " examinatoire ${ }^{19}$ " pour reprendre le vocabulaire et la démonstration de Michel Foucault dans Surveiller et punir). Rien qu'aux États-Unis, on compte plus de 40 clinics en $1933^{20}$. Ces services vont étendre progressivement leur clientèle au-delà des jeunes délinquants pour comprendre, soigner et réparer le psychisme de l'enfant, la justice des mineurs ouvrant une voie au contrôle plus global des populations par le Welfare State.

Dernière pièce essentielle dans le montage juridique des TE: la probation, qui permet d'éviter l'enfermement. Elle permet aussi au juge des enfants de garder la main sur le cas en ne prononçant pas de décision définitive de place- 
ment, puisque la Constitution du Massachusetts garantit une stricte séparation des pouvoirs, à la différence du cadre constitutionnel français: si le juge des enfants envoie un mineur en training school ${ }^{21}$, il a rendu sa décision et ne peut plus intervenir ensuite, c'est l'Éxécutif, à travers le Conseil d'administration des training schools, transformé en agence publique par une loi d'État de 1948, à partir d'un modèle posé en 1940 au niveau fédéral ${ }^{22}$, qui prend les mesures ultérieures - en particulier la mise en liberté conditionnelle une fois le mineur " amélioré ». Le principe posé avec force par le législateur français, en 1945, d'un suivi continu par l'autorité judiciaire et du " caractère essentiellement révisable " de ses décisions dans " l'intérêt de l'enfant ${ }^{23}$ " se heurte aux États-Unis même, qui ont inspiré cette logique, au principe général des checks and balances (poids et contrepoids), faisant surgir un des points de tension entre les droits des citoyens, leur protection constitutionnelle, l'organisation démocratique et les particularités et besoins de l'enfance et de l'adolescence. Si le pouvoir arrête le pouvoir, la justice des mineurs, qui en a relativement peu, semble plus encline dans la configuration américaine à se retrouver prise en tenaille par le pouvoir exécutif, entre la police et les agences de placement. La probation peut alors servir, non seulement à garantir la vocation éducative du tribunal, mais aussi de technique légale pour résister à d'éventuelles tendances répressives de l'Éxécutif.
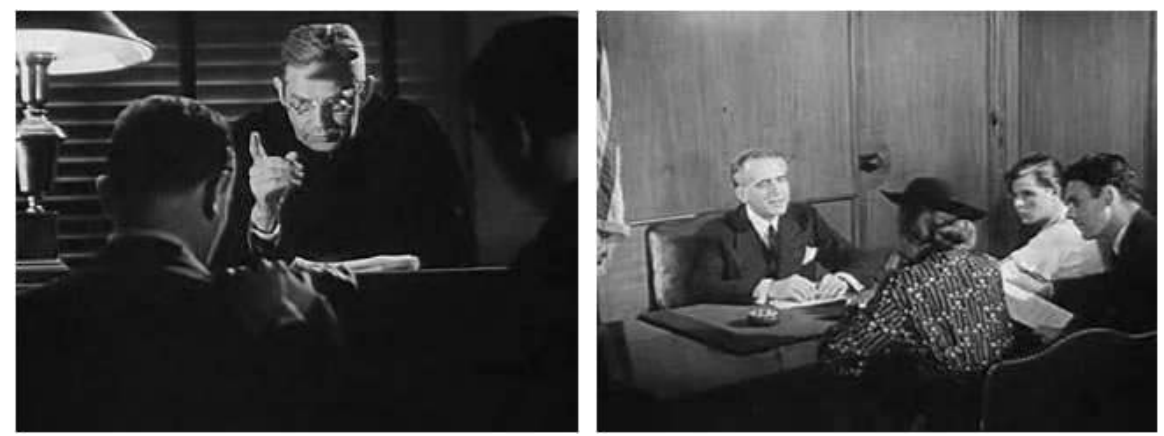

Illustrations 1 et 2. Du tribunal répressif au tribunal pour enfants. Source: Lion David H. (director), Kienle Edward C. (producer [supervisor]) and Furman Robert T. Jr. (writer), Boy in Court, Sponsor: National Probation Association, Production Co. : Willard Pictures Inc., 1940, $2 \mathrm{~min} 22 \mathrm{~s}$ et $5 \mathrm{~min} 43 \mathrm{~s}$. Disponible sur: [http://archive.org/details/BoyinCou1940] (consulté le 26 mai 2012).
21. Les training schools (littéralement: écoles de formation) désignent, par euphémisme, des centres fermés (décrits d'ailleurs comme des institutions

" ouvertes", entre guillemets dans le texte, dans Massachusetts, Division of Youth Service, Service to Youth: "The Story of the Youth Service Board", [Boston], 1 March 1955, p. 10-13). II existe, dans le Massachusetts, deux " écoles " pour les garçons et une pour les filles.

22. En 1940, l'American Law Institute publie un projet de Youth Correction Authority Act, proposant un modèle d'organisation (une « autorité ") pour le traitement correctionnel des jeunes (de 16 à 21 ans selon le projet initial).

23. Exposé des motifs de: France, Ordonnance relative à l'enfance délinquante. 2 février 1945. 
24. LIon David H. (director) KIENLE Edward C. (producer

[supervisor]) and FURMAN Robert T. Jr. (writer), Boy in

Court, Sponsor: National Probation Association,

Production Co. : Willard

Pictures Inc., 1940, 10 min $26 \mathrm{~s}$. Disponible sur: [http://archive.org/details/ BoyinCou1940] (consulté le 26 mai 2012)

25. JULHIET, « Les tribunaux pour enfants... », op. cit.

p. 58

26. Discours introductif de W. P. C. Knuttel, juge des enfants à Amsterdam,

au congrès de 1950 de l'Association internationale des juges des enfants, cité dans CHarvin Monique et al.,

Recherches sur les juges des enfants: approches historique, démographique, sociologique: rapport final et annexes, Paris, ministère de la Justice, 1996, p. 107. Disponible sur: [http://www. ladocumentationfrancaise.fr/ rapports-publics/984000445/ index.shtml\#book_sample] (consulté le 11 février 2013).

27. JULHIET, « Les tribunaux pour enfants... », op. cit.,

p. 62.
Le film Boy in Court (Le garçon au tribunal) ${ }^{24}$ de 1940 fait en 10 minutes la publicité des agents de probation (probation officers) dans les TE, vantant leur dévouement et leur professionnalisme pour mieux marquer la rupture d'avec le temps des bénévoles. Le spectateur visualise immédiatement le passage de l'ombre à la lumière (ill. 1 et 2) : à la salle d'audience sinistre d'un tribunal répressif pour les adultes et les vrais criminels, pointés d'un doigt accusateur, succède le cabinet moderne, confortable et bien éclairé d'un juge des enfants.

Un dialogue compréhensif peut s'engager. Plus besoin de procureur ou d'avocat, puisque le juge est «le défenseur naturel de l'enfant ${ }^{25}$ " et de ses intérêts supérieurs. Autour du bureau se trouvent quatre personnages: le jeune délinquant, sa mère, l'agent de probation (présent dès le départ), enfin le juge, en habit de ville, " descendu de son piédestal ${ }^{26}$ ", pour reprendre une expression dénotant l'engouement de plus en plus grand chez les professionnels de l'enfance pour ces nouvelles procédures, plus humaines. Le film montre ensuite, en opposition à la solution carcérale, la rééducation et la réintégration du mineur dans la communauté sous la surveillance et avec l'aide de l'agent de probation, qui établit des " relations amicales et utiles ${ }^{27}$ " après avoir mené une enquête approfondie sur le jeune et son milieu. Le résultat est visible à l'écran (ill. 3 à 6). Avant: un délinquant débraillé, en T-shirt, fomentant la nuit de mauvais coups avec sa bande. Après: un jeune en cravate, presque cloné sur son agent de probation dont il a revêtu le costume croisé.
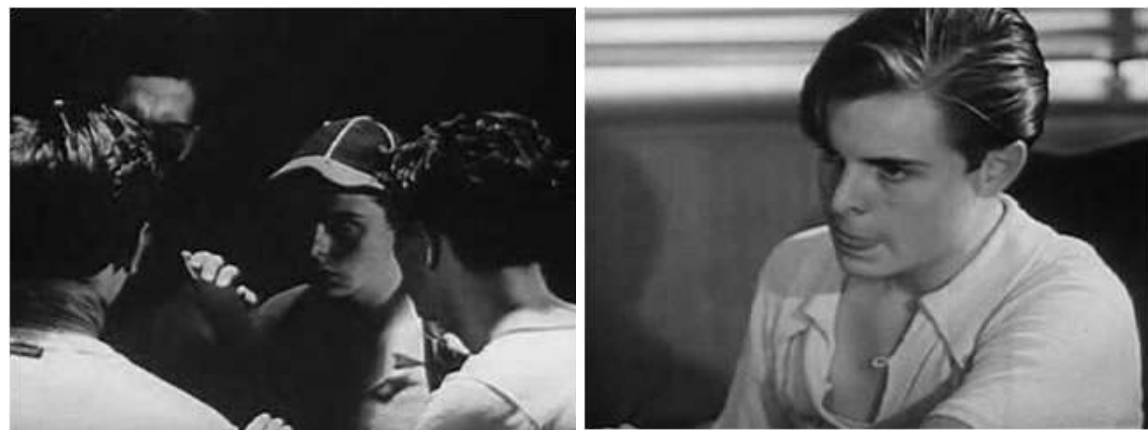

Illustrations 3 et 4 . AVANT la probation. Source: Lion, Kienle and Furman, Boy in Court..., $54 \mathrm{~s}, 6 \min 6 \mathrm{~s}, 9 \mathrm{~min} 28 \mathrm{~s}$ et $9 \min 38 \mathrm{~s}$. 

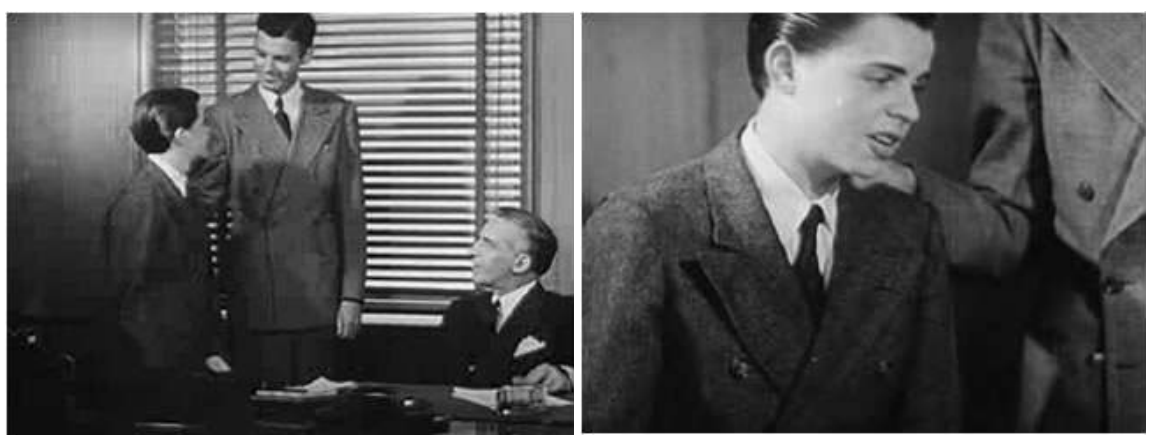

Illustrations 5 et 6 . APRÈS la probation. Source: Lion, Kienle and Furman, Boy in Court..., $54 \mathrm{~s}, 6 \mathrm{~min} 6 \mathrm{~s}, 9 \mathrm{~min} 28 \mathrm{~s}$ et $9 \min 38 \mathrm{~s}$.

Cette propagande renvoie à l'importance réelle du service de probation dans les tribunaux américains. Dans les juvenile courts, le juge travaille très étroitement avec l'agent de probation (souvent une femme pour les jeunes filles) en amont et en aval de la décision de mise en liberté surveillée. D'ailleurs, les dossiers individuels de la BJC, conservés pour les années 1940 et 1950, sont intitulés des "probation files" (dossiers de probation). Des pratiques actuelles peut-on déduire que le juge des enfants se présente à l'audience sans véritable dossier propre, le dossier du mineur ayant été préparé par le service de probation, qui en assure le suivi. Peut-être faut-il y voir, entre autres, l'héritage du système accusatoire où le juge, arbitre entre les parties, doit entrer au tribunal sans préjugé sur l'affaire.

Il n'y a pas d'équivalent exact au probation officer dans le système français. On ne peut le comparer ni à l'assistante sociale, ni au délégué à la liberté surveillée, qui n'intervient qu'après-coup, ni au greffier, dont le rôle de collaborateur a pu être souligné28. Le long-métrage Chiens perdus sans collier, sorti en 1955, insiste sur la relation personnelle du juge avec le mineur, dont l'exclusivité peut se trouver accentuée par une certaine centralisation des procédures françaises autour du magistrat - les pratiques et le caractère du juge restant fondamentaux. L'une des affiches du film (ill. 7) montre le personnage du juge Lamy, incarné par un Gabin tendre et bourru, la main posée sur l'épaule de l'enfant, rapport physique exprimant attention, bienveillance et confiance, revenant comme un symbole récurrent d'altruisme dans les représentations et les discours sur les TE de part et d'autre de l'Atlantique ${ }^{29}$.
28. Charvin Monique et al., Recherches sur les juges des enfants..., p. $173 \mathrm{du}$ PDF.

29. Par exemple, pour rester dans le domaine des images, voir l'emploi qu'en a fait le juge pour enfants de Denver, Lindsey: petite fille sur les genoux, etc. Certaines de ses photographies sont disponibles sur le site de la Bibliothèque du Congrès; ainsi, celle, éloquente, où le célèbre juge à moustache fait face à un gamin et à son chien posé sur le bureau, les désignant d'un doigt ne symbolisant plus que la sévérité du bon père de famille, sinon une certaine connivence dans la mise en scène: [http://www.loc.gov/ pictures/item/2002710146/] (consulté le 10 septembre 2013). 
30. Voir le docteur Bronner photographiée à la Judge Baker Foundation, la main sur l'épaule de l'enfant qu'elle teste: [http://jbcc.harvard.edu/ sites/default/files/styles/ photogall_zoom/public/ galleries/dr-bronner-andchild.jpg?itok=yP1gfYnS] (consulté le 9/8/2013).

31. Rappelons l'importance, au commencement des TE, des audiences publiques pour légitimer l'institution et communiquer sur le rôle des juges des enfants.

32. Des bains et douches sont d'ailleurs donnés dans

le TE de Denver dans les années 1900 (Les tribunaux spéciaux..., p. 136 et p. 138).

33. Je reprends les termes employés à l'occasion d'un procès, en 1907, par une chambre de commerce "forcée " de déménager suite à l'arrivée dans l'immeuble du TE de Chicago,

avec ses populations

« indésirables », souvent immigrées. Cité dans Tanenhaus David S., Juvenile Justice in the Making, New York, Oxford University Press, 2004, p. 27-28.

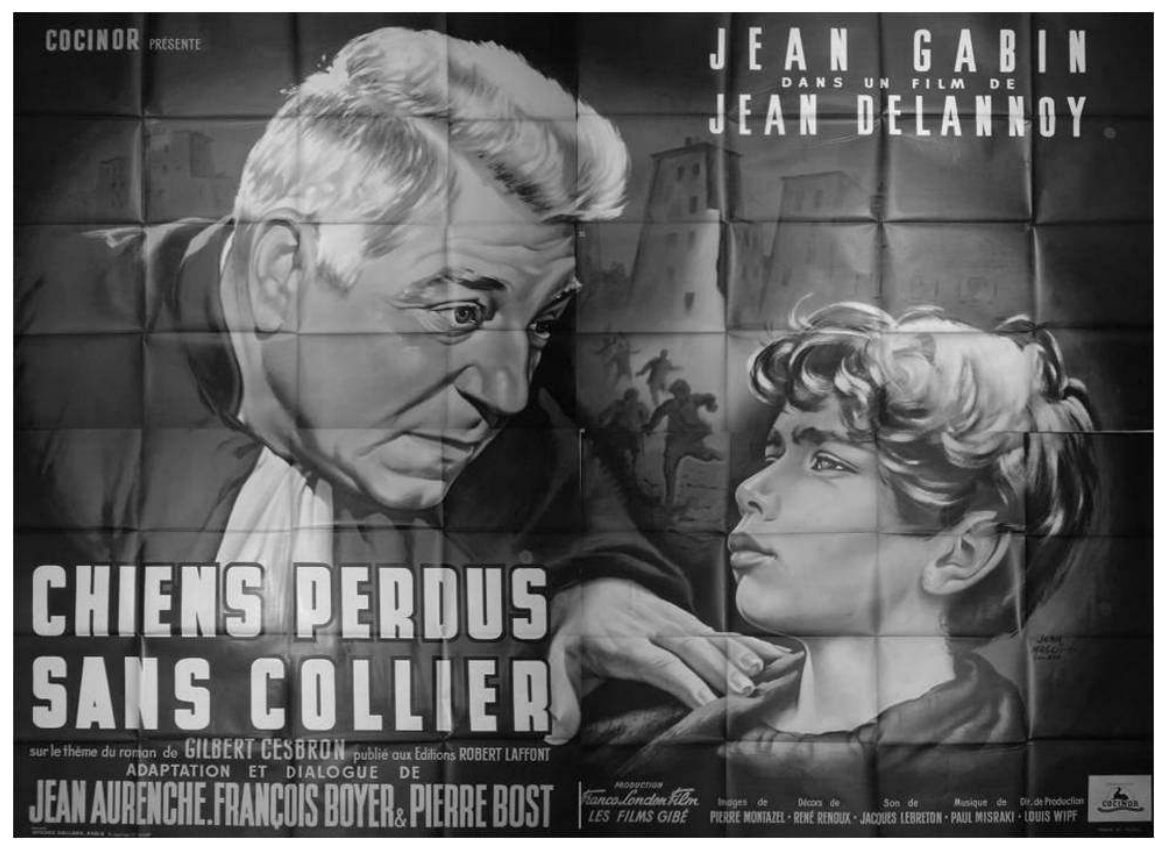

Illustration 7. Affiche publicitaire du film Chiens perdus sans collier réalisé par Jean Delannoy, d'après le roman de Gilbert Cesbron, et sorti en 1955. Source : affiche disponible, par exemple, sur: http://cinemapalaceequeurdrevillelesannees50.blogspot.fr/2010/03/programme-du-moisde-mai-1956.html (consulté le 26 août 2013).

Ce topos s'oppose au doigt accusateur du juge répressif, qui domine le suspect et le tient à distance; il se décline dans les relations mineurs/agents de probation (ill. 5 et 6 ), mineurs/psychologues ${ }^{30}$, etc. Il faut voir dans ce geste plus qu'une donnée publicitaire, liée à la nécessité de vendre les juvenile courts ou leur pendant fictionnel auprès du public ${ }^{31}$, et considérer les acteurs dans leur sincérité même, dont la signification les dépasse, car elle s'inscrit dans l'évolution des mœurs et du ressenti social. Au tournant du $\mathrm{xx}^{\mathrm{e}}$ siècle, lorsque se créent les premiers TE, le contact physique entre un bourgeois (le juge) et un enfant du peuple (le délinquant), parfois misérable, sale et malodorant ${ }^{32}$, et même " affligé de maladies ", " mauvais ", " dépravé ", "vicieux ${ }^{33}$ "... est à interpréter comme un outrepassement des frontières de classes, la reconnaissance d'une commune humanité, qu'encourage probablement le sentiment de l'enfance, puis de l'adolescence. 
Tocqueville notait que, dans les siècles aristocratiques, "la notion générale du semblable est obscure ${ }^{34}$ " alors que dans les démocraties, et aux États-Unis en premier lieu (à l'époque de son enquête, dans les années 1830), le mouvement d'égalisation des conditions ${ }^{35}$ favorise la "sympathie " et la "compassion " entre êtres humains. Les TE s'inscrivent dans ce mouvement général de montée, puis de renforcement de la démocratie, où «l'on n'accorde guère de grands bienfaits ", mais " rend sans cesse de bons offices ${ }^{36}$ ", car, si les liens entre les hommes se sont affaiblis, le cercle de ses semblables s'est élargi. Les gestes, positions corporelles, etc., des juges des enfants et autres intervenants expriment sans doute cette sensibilité démocratique plus aiguë, qui ne permet plus, ou plus aussi facilement, de classer les pauvres, et leur progéniture, dans un groupe à part ou une espèce distincte.

De la procédure inquisitoire française reste toujours l'instruction, ignorée du système accusatoire américain, qui repose sur les procédures orales (nul greffier, d'ailleurs, dans le cabinet du juge de Boy in Court). Aux États-Unis, la preuve est essentiellement faite à l'audience et l'on chercherait en vain, dans les dossiers de la BJC, des rapports de police équivalents aux PV français. Lors de l'audience, le témoignage direct du policier les remplace. Ajoutons que la culture juridique américaine favorise l'aveu et que le mineur n'est pas habituellement représenté par un avocat, alors que l'on observe dans les dossiers conservés du TE de la Seine (postérieurs à l'ordonnance de 1945) la généralisation des commissions d'office, même si l'avocat reste bien souvent un " acteur muet ${ }^{37}$ ", voire absent lorsqu'il s'abstient de paraître à l'audience...

Les États-Unis sont donc allés plus loin que la France dans la rupture avec le système pénal et avec les formes juridiques du procès équitable. Le juge des enfants américain n'est plus l'arbitre d'une lutte entre les parties d'où émergerait la vérité judiciaire, selon les théories de common law; il n'y a plus de lutte, car il n'y a plus de parties, tout est régi par l'idéologie de l'intérêt de l'enfant. La France semble adopter une position plus modérée, maintenant l'instruction même si elle est normalement menée par le juge du jugement en contradiction avec les principes généraux de la procédure pénale. La Revue de l'Éducation Surveillée, qui paraît en 1946, possède une rubrique juridique, où sont abordés des problèmes de procédure et de droit du mineur parfois très techniques. Aux États-Unis, au contraire, l'informel a pu être érigé en règle de bonne justice,

\author{
34. TOCQueVILle Alexis de, De \\ la démocratie en Amérique, \\ version parue en 1840 , \\ Textes essentiels: anthologie \\ critique, présentée par Jean- \\ Louis Benoît, Paris, Pocket, \\ Agora, 2000, p. 98. \\ 35. Tocqueville souligne que \\ la manière très dure dont \\ sont traités les Noirs améri- \\ cains, exclus de la citoyen- \\ neté et réduits à l'esclavage, \\ confirme sa théorie: plus \\ que la civilisation, c'est bien \\ la mécanique démocratique \\ d'égalisation qui adoucit les \\ mœurs, car elle fait craindre \\ à tous les citoyens les \\ mêmes maux, provoquant \\ une entraide conforme à \\ l'intérêt de chacun et au \\ sentiment de sympathie qu'il \\ porte à son « semblable ». \\ 36. Tocqueville Alexis de, De \\ la démocratie en Amérique, \\ version parue en 1840 , \\ Textes essentiels..., p. 107 \\ 37. Benec'h-Le Roux \\ Patricia, « Les avocats dans \\ les tribunaux pour enfants: \\ des acteurs longtemps \\ muets de la justice depuis \\ 1890 », RHEI, n 12, 2010 , \\ p. 87-109.
}


38. Bernard Thomas J. and KurLYCheK Megan C., The Cycle of Juvenile Justice, $2^{\text {nd }}$ edition, New York, Oxford University Press, 2010, p. 80.

39. Voir les débats juridiques autour de la loi de 1912: Charvin Monique, Gazeau Jean-François et al., Recherches sur les juges des enfants..., p. 24-28.

40. The Citizenship Training Group, Inc., Research Department (compiler and ed.), Boston Juvenile Court: Annual Report, 1951, 28 p.

41. Mérat Juan, Les enfants devant le juge, 1945-1958: les stratégies normatives au sein d'un cabinet du Tribunal pour enfants de la Seine, Maîtrise, Histoire, sous la dir. de Danielle Tartakowsky,
Paris 8, 2001, 168p. et annexe de $79 p$.

42. Le rapport de la Boston Juvenile Court intègre dans ces 567 affaires de "delinquency":

27 affaires de stubborn children (enfants têtus), qui pourraient être comparées aux affaires françaises

de correction paternelle (celles-ci ne relevant pas de la " délinquance ") ou, peutêtre plus encore, aux affaires de vagabondage, dépénalisé en 1935, mais toujours considéré, en pratique, comme de la délinquance ou de la prédélinquance; 5 affaires d'enfants indisciplinés (wayward children), qui ne sont pas juridiquement des délinquants, mais s'en rapprochent du point de vue des procédures effectivement suivies par le tribunal. survalorisé au point de sembler transformer la juvenile court en "coercive casework agency ${ }^{38}$ " (service social coercitif).

On a pu souligner la nouveauté dans le système pénal français de la loi de 1912, mais le TE est également une nouveauté dans le système pénal américain lorsqu'il y est introduit. Même l'institution du magistrat unique, si décriée par les partisans de la tradition judiciaire française, désirant limiter l'arbitraire par la collégialité des décisions ${ }^{39}$, ne s'inscrit pas plus dans la coutume américaine à partir du moment où sa nature d'arbitre disparaît. En revanche, la probation est une pratique qui, partie de Boston dans les années 1840, s'est déjà répandue aux États-Unis lorsque les instigateurs des TE américains la récupère pour éviter l'enfermement des mineurs qu'ils souhaitent prendre en charge.

\section{LES POPULATIONS VISÉES \\ (L'EXEMPLE DES ANNÉES 1950)}

À partir des chiffres de 1951 produits par le TE de Boston ${ }^{40}$ et des statistiques construites a posteriori pour un cabinet du TE de la Seine pour la période allant d'octobre 1956 à novembre $1958^{41}$, essayons de déterminer les principales caractéristiques de la clientèle visée en ce milieu du $\mathrm{xx}^{\mathrm{e}}$ siècle par la justice des mineurs.

À Boston, on compte, pour une année, 567 affaires de délinquance juvénile ${ }^{42}$, mettant en cause 486 mineurs, auxquelles il faut ajouter 14 affaires de négligence d'enfants, affectant 47 mineurs, et une cinquantaine d'affaires concernant des majeurs pour des faits de négligence d'enfants 
ou de contribution à la délinquance ou à l'indiscipline d'un mineur. C'est l'équivalent en gros de l'activité de deux cabinets du TE de la Seine, qui en compte $\operatorname{six}^{43}$. Quelques ordres de grandeur pour les affaires de délinquance juvénile ${ }^{44}$ :

- À Boston, comme dans le département de la Seine, plus de la moitié des affaires, autour de 55 à $60 \%$, concernent des vols et atteintes aux biens. L'autre grande catégorie, environ un cinquième à un quart des affaires, est une catégorie attrape-tout, depuis la prostitution jusqu'aux comportements jugés immoraux ou dangereux pour l'équilibre social ou familial; elle correspond, dans la Seine, au vagabondage, qu'il soit réel ou prétexte à l'intervention, et à l'addition des catégories américaines de runaway (fugueurs) et stubborn children (enfants têtus), auxquelles on peut ajouter les cinq affaires de wayward children (enfants indisciplinés), voire les affaires de truancy (absentéisme scolaire), status offenses ${ }^{45}$ dont les pénalistes français se sont toujours méfiés, mais qui sont peu nombreuses (douze), alors qu'elles se chiffraient à près d'une centaine lors de la première année de fonctionnement du tribunal ${ }^{46}$. Derrière des définitions juridiques différentes, qui compliquent toute comparaison, se retrouvent grosso modo les mêmes grandes structures des illégalismes juvéniles, comme si, après les premières phases d'expérimentation, et en lien avec les évolutions sociales, culturelle et économiques, les pratiques des TE (dans des villes de taille importante) semblaient se rapprocher.

- Concernant l'âge, la majorité pénale est fixée à 17 ans dans le Massachusetts et à 18 ans en France; en dessous de 7 ans, en raison de la loi ou de la tradition, il n'y a pas de poursuite. Les prévenus sont très majoritairement des adolescents: entre 80 et $90 \%$ de 14-17 ans pour la Seine et de 13-16 ans pour Boston, chaque institution semblant s'adapter à la frontière de la majorité pénale, sauf à supposer que le délinquant américain soit un peu plus précoce.

- Sur la question du genre, dans la Seine, la proportion garçons/filles est très stable dans le temps, environ une fille pour quatre à cinq garçons; à Boston, la parité n'est pas très loin ( $40 \%$ de filles). Ce pourcentage élevé, en comparaison des résultats habituellement disponibles, ne se retrouve pas dans le reste du Massachusetts et serait dû, selon le TE de Boston, à la présence des grands magasins dans le centre-ville, où s'étend sa juridiction, qui attireraient les adolescentes venues y pratiquer le vol à l'étalage ${ }^{47}$.

Tout un travail de production et de croisement des données reste à faire, par exemple pour affiner la question de l'origine sociale, généralement populaire,
43. Selon mon dénombrement des dossiers du TE de la Seine pour 1950.

44. On y intègre les affaires françaises de vagabondage pour les raisons données en note 42.

45. Actes illicites du fait qu'ils sont commis par un mineur.

46. LENROOT Katharine F. and LundBERg Emma O., Juvenile Courts..., p. 241.

47. Boston Juvenile Court: Annual Report..., p. 17. 
48. Ce qui n'empêche pas certains intervenants

(assistantes sociales, etc.) de faire occasionnellement des remarques à connotation raciale ou raciste. L'approche et la compréhension de la société française restent cependant très influencées par l'idée abstraite de communauté

nationale et la réticence

à accorder un rôle aux groupes et communautés intermédiaires.

49. Parmi les premiers exemples de cette approche culturaliste: The Spirit of Youth and the City Streets de Jane Adams (1909) et The Delinquent Child and the Home de Sophonisba

P. Breckinridge et Edith Abbot (1912). Tous deux disponibles sur le site [www. archive.org]

50. Archives du Department of Youth Services du Massachusetts, File C [remarque: nous respectons les instructions de chaque institution concernant le respect de l'anonymat des individus mis en cause (changement des noms, etc.)].

51. Voir entre autres JacoBson Matthew Frye, Whiteness of a Different Color: European Immigrants and the Alchemy of Race, Cambridge, Mass., Harvard University Press, 1998, $\mathrm{X}-338 \mathrm{p}$.

52. Archives de la Massachusetts Supreme Judicial Court (MSJC), BJC Samples, Box 2, Probation File 2980. des jeunes délinquants, ou comprendre la formation des groupes. Les manières de classer sont elles-mêmes à étudier: le classement trop évident par genre, dont la naturalisation soutient la domination du mâle hétérosexuel et justifie le contrôle minutieux de la sexualité féminine, mais également les classements par quotient intellectuel, par régularité de la fréquentation religieuse, etc., qui renvoient à des savoirs et à des (di)visions ordonnées du monde social partagés ou propres à chaque pays.

Ainsi, à la différence des dossiers français, où toute mention de race est interdite, l'État-nation ne distinguant que les citoyens des non-citoyens, générant une myopie officielle face à cette question ${ }^{48}$, les dossiers américains peuvent employer des catégories raciales ou ethniques. Elles servent à relier le mineur à une communauté, soutien et structure de l'individu dans un immense État fédéral conçu comme une République où les minorités sont protégées et reconnues. Mais, à travers cette approche identitaire, se pose aussi la question de l'américanisation, dans une logique souvent très WASP (selon la norme du "Blanc, anglo-saxon et protestant ", d'autant plus prégnante que l'on se trouve en Nouvelle-Angleterre), avec l'objectif, au moins, de prévenir les conflits culturels, chaque communauté devant respecter l'autre et respecter les valeurs du nouveau monde ${ }^{49}$. John, par exemple, est né aux États-Unis, mais la nationalité indiquée dans son dossier de training school ${ }^{50}$, où il est placé en 1944, est française, car ses parents sont des Canadiens francophones. Se présentent également des intitulés mixtes, franco-italien par exemple, rappelant que les relations intercommunautaires peuvent pousser vers l'absurde ce type de classement, s'appuyant in fine sur la notion de race pour constituer la grille de lecture d'un monde social où les immigrés de deuxième, troisième... générations sont de plus en plus nombreux et de moins en moins classables dans les limites d'une communauté culturellement définie. D'où également la tentative de relier le processus de progression dans l'américanisation à des degrés de "blancheur " de peau (whiteness) ${ }^{51}$ : une mère de famille, pourtant née et mariée à Boston, est de " couleur olive s2 $^{2}$, d'un blanc douteux en somme, en raison de ses origines italiennes et, sans doute, de son inadaptation relative aux mœurs américaines vu son abondante progéniture (neuf enfants).

Les Noirs sont peu nombreux à Boston (moins de $5 \%$ de la population), même s'ils se concentrent dans certains quartiers plus ou moins ghettoïsés. Globalement, si dans le nord des États-Unis, l'approche raciale peut générer 
une ségrégation de fait ${ }^{53}$, dans le sud elle entraîne une ségrégation légale, qui se perpétue dans les institutions judiciaires, lieux de placement, etc. ${ }^{54}$.

Un dernier exemple d'écart entre les jeunes délinquants français et américains : la manière dont ils sont ciblés médiatiquement. Des coupures de journaux apparaissent assez régulièrement dans les probation files, révélant comment est résolue aux États-Unis la tension entre liberté de la presse, présomption d'innocence et protection des mineurs (que l'anonymat et le secret des procédures sont censés assurer).

La culture américaine est peu favorable au secret. Elle valorise la transparence et le pouvoir des médias, exigeant de l'individu qu'il assume ses actes face à la communauté, au-delà de la réponse judiciaire. L'affaire Strauss-Kahn de 2011 a mis ces éléments de pouvoir (et de violence corrélative) en exergue, choquant une partie de l'opinion française à la diffusion des images d'une personnalité politique qui, dès les premières accusations, se retrouvait menottée, emprisonnée, etc. ${ }^{55}$. Plus modestement, Frank ${ }^{56}, 16$ ans, au casier judiciaire bien rempli, fait l'objet d'un article dans la presse locale des années 1950. Est reproduite sa photo (ill. 9), sont donnés son nom et son adresse. L'affaire contient les ingrédients du crime à l'américaine, aptes à satisfaire un public friand de faits divers: un jeune gangster, et sa bande, qui, au volant d'une voiture volée, heurtent un arbre après une course poursuite avec la police; des holdups dans des stations
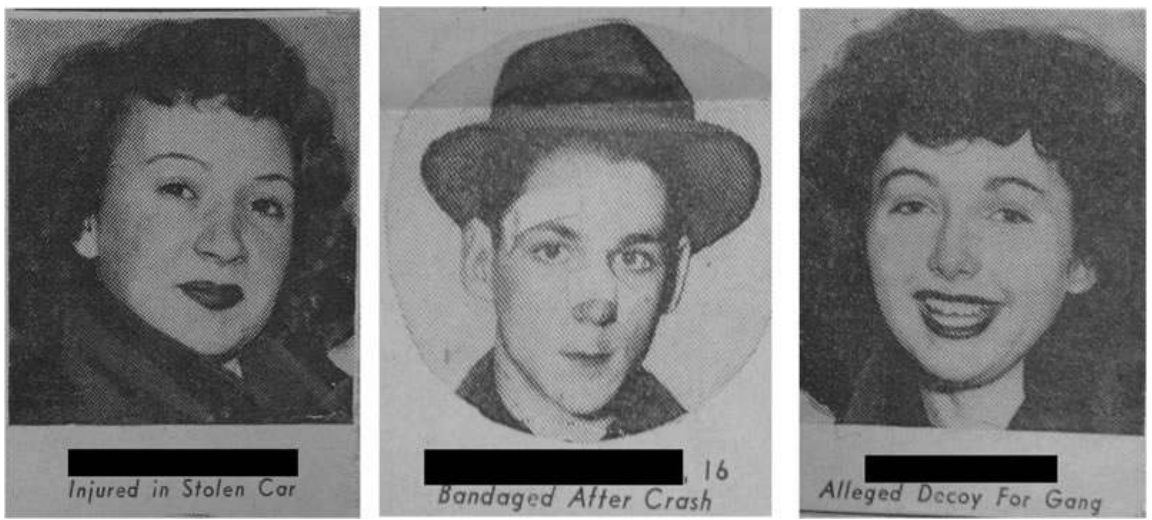

Illustrations 8, 9 et 10. Un jeune « holdup man » (braqueur) de 16 ans, avec deux de ses complices: « une incroyable carrière dans le crime ». Source: article découpé d'un journal de Boston des années 1950 [anonymisé par l'auteur], Archives de la Massachusetts Supreme Judicial Court, BJC Samples, Box 1, Probation File 20.
53. Utilisant les dossiers individuels de la Judge Baker Foundation: COREA Joseph Carlo, Racial Delinquency: Italian-American and African-American Adolescent Identity and the Delinquency Experience, 1945-1932, Ph. D. thesis, History, advised by Matthew F. Jacobson, State University of New York at Stony Brook, May 2001, V-211p.

54. Ward Geoff K., The Black Child-Savers: Racial Democracy and Juvenile Justice, Chicago, The University of Chicago Press, 2012, 352p.

55. Voir Garapon Antoine et Villez Barbara, « Retour sur l'affaire DSK: un éclairage culturel ", Point de vue sur le site de l'Institut des Hautes Études sur la Justice, 16 décembre 2011. Disponible sur: [http://www. inej.org/retour-sur-laffairedsk-un-eclairage-culturel/] (consulté le 28 février 2013).

56. Archives de la MSJC BJC Samples, Box 1, Probation File 20. 
57. Par exemple, la déclaration d'Arthur E. Paul, président du Massachusetts Council of Juvenile Probation Officers, dans United States, Congress, Senate, Committee on the Judiciary, Juvenile Delinquency (Boston,

Mass.): Hearings Before the Subcommittee to Investigate Juvenile Delinquency..., 28, 29 and 30 January 1954 , Washington, United States Government Printing Office, 1954, p. 139 sq

58. Nous nous appuyons sur: Charvin Monique, GazeAU Jean-François et al., Recherches sur les juges des enfants... ; FARCY Jean-Claude (réalisation de la base de données) et FRY Rosine (programmation et portage web),
Annuaire rétrospectif de la magistrature $X I x^{e}-x x^{e}$ siècles, Centre Georges Chevrier (université de Bourgogne/ CNRS), mis en ligne le 12 juin 2010. Disponible sur: [http://tristan.u-bourgogne. fr/AM.html] (consulté le 3 avril 2013). Sur la carrière de Robert Chadefaux, voir en particulier (une partie du discours étant consacrée à ce magistrat, mort le 24 février 1966): SchmELCK Maurice (avocat général à la Cour de cassation), Discours prononcé lors de

l'audience solennelle de rentrée, le 3 octobre 1966. Disponible sur: [http:// www.courdecassation.fr/ institution 1/occasion audiences_59/but_ann_60/ ann es 1960 3337/ octobre_1966_10406.html] (consulté le 3 avril 2013). d'essence, avec armes à feu et jeunes filles servant de couverture (ill. 8 et 10); une vie sexuelle et amoureuse précoce, Frank ayant mis enceinte sa petite amie, avec qui il vit maritalement sous de faux noms. La petite amie conservait d'ailleurs les journaux relatant les exploits de son "époux ». Aussi cette publicité faite à de jeunes délinquants, leur permettant d'accéder à une certaine notoriété, est-elle parfois dénoncée comme le motif même de la déviance ${ }^{57}$. Prochaine étape pour Franck et ses comparses: le tribunal pour y être jugés.

\section{LES JUGES ET LEURS JUGEMENTS}

Les juges sont en général des hommes (en France, la magistrature est interdite aux femmes jusqu'en 1946) qui ont suivi une formation juridique, mais leur sélection, leur carrière et leur environnement professionnel sont bien différents dans les deux pays.

Prenons, pour la France ${ }^{58}$, le profil relativement typique d'un des premiers juges pour enfants, ayant mené une belle carrière. Robert Chadefaux, né en 1898 dans une famille de magistrats, décroche un doctorat en droit et devient juge suppléant, substitut, puis procureur. En 1934, il est nommé juge d'instruction adjoint au tribunal de la Seine, où il est rapidement affecté à la section des mineurs. Pendant la guerre, il accède à la position de président du TE de la Seine, qu'il occupe jusqu'en 1953, lorsqu'il est promu président de chambre, finissant conseiller à la Cour de cassation.

Aux États-Unis, les magistrats se distinguent des serviteurs de l'État à la française par la proximité d'avec les avocats, évoluant dans le même monde des lawyers (hommes de loi). Le TE de 
Boston est organisé avec un président, assisté de deux juges spéciaux qui travaillent à temps partiel et continuent des carrières d'avocat. Afin d'assurer leur indépendance, ils sont nommés à vie par le gouverneur, avec accord de son conseil, ceci pouvant entraîner des tractations politiques.

La pratique est de respecter un équilibre dans la représentation des principales communautés de Boston. En 1906, le trio de magistrats est constitué par: un président WASP, le juge Baker, «a thorough New Englander ${ }^{59}$ " (profondément de la Nouvelle-Angleterre), diplômé d'Harvard et membre actif de l'Église unitarienne; le juge spécial Rubenstein, russe et juif; et le juge spécial Leveroni, italien et catholique. À la fin de la période étudiée, en 1950, on trouve: un président irlandais et catholique, le juge Connelly; le juge spécial Rubenstein, qui est toujours là, probablement septuagénaire; et un juge noir, le juge Robinson, deuxième juge afro-américain de l'histoire du Massachusetts.

Il semble que la présidence du TE de Boston doive revenir à l'une des deux communautés dominant la ville. Les premiers présidents sont WASP, appartenant à l' " aristocratie » bostonienne des brahmins ${ }^{60}$, qui trouvent dans la philanthropie et la participation au gouvernement (de la justice) certaines raisons d'être et certaines formes légitimes et légitimantes d'exercice du pouvoir. En 1945 est désigné le quatrième président de la BJC. Cette fois, il est irlandais et catholique. Indépendamment de l'évolution des rapports de force au sein des organes de nomination, le choix du juge Connelly est sans doute rendu possible par le moindre attrait de la position de président, au niveau symbolique du moins, par rapport à la grande époque de l'idéal des juvenile courts. D'après les informations trouvées, John J. Connelly a le parcours d'un homme de la middleclass. Né et ayant grandi dans le quartier de Dorchester, connu pour ses petits immeubles bon marché en bois (triple-decker) accueillant une forte population immigrée, il sort diplômé en droit du Boston College, université catholique, où il était une "star du baseball ${ }^{61}$ ", poursuivant une carrière d'agent de probation auprès des mineurs et gravissant les échelons de la hiérarchie du juvenile justice system bostonien.

Chaque juge est souvent actif dans sa communauté, qu'il représente en quelque sorte. Ainsi, le juge Rubenstein travaille avec les associations juives, qui le considèrent comme un allié au tribunal ${ }^{62}$, pour éviter, par exemple, le placement d'enfants israélites dans des institutions ou foyers chrétiens où ils risqueraient de perdre leur identité - l'intégration sociale passant par la commu-

59. Cushman Roy M., "Harvey Humphrey Baker: Man and Judge", Harvey Humphrey Baker: Upbuilder of the Juvenile Court, Boston, Mass., Judge Baker Foundation, [1920], p. 2. Disponible sur: [http:// www.archive.org/details/ harveyhumphreyba00judg] (consulté le 26 mars 2013).

60. Les brahmins, en référence à la caste supérieure des brahmanes en Inde, constituent l'élite WASP traditionnelle, aisée, cultivée et cultivant des formes aristocratiques de désintéressement et de dévouement à la communauté. Dans Holloran Peter C., Boston's Wayward Children: Social Services for Homeless Children, 1830-1930, Rutherford [N.J.], Fairleigh Dickinson University Presses, London and Toronto, Associated University Presses, 1989 , 330p., l'auteur insiste sur l'appartenance à cet « establishment " des premiers juges de la BJC.

61. "B. C. Alumnus State's Fourth Juvenile Judge ", The Heights [journal des étudiants du Boston College], vol. 26, $n^{\circ} 7$, 19 December 1945, p. 4. Disponible sur: [http://newspapers.bc.edu/cgi-bin/bost onsh? $\mathrm{a}=\mathrm{d} \& \mathrm{~d}=$ bcheights 19 451219.1.4\&e=-------en-20-1--txt-IN-----] (consulté le 5 septembre 2013).

62. Holloran Peter C., Boston's Wayward Children..., p. 170 sq. 
63. Voir, par exemple, le douloureux parcours dans les institutions du Massachusetts, depuis l'âge de sept ans, d'un «stubborn child ", né en 1948, dans l'autobiographie: DEvLIN Mark, Stubborn Child, New York, Atheneum, 1985, 255 p.

64. Boston Juvenile Court: Annual Report..., p. 21. On a additionné les catégories de «probation » et de « probation and suspended sentence " (probation et sursis).

65. L'expression revient régulièrement dans: LENROOT Katharine $F$. and LUNDBERG Emma O., Juvenile Courts...

66. Archives de la MSJC, BJC Samples, Box 1, Probation File 1.

67. Archives de la MSJC, BJC Samples, Box 1, Probation File 260.

68. Évangile de Luc cité dans Connelly John J., "Children are not expendable ", 24 September 1952, reprinted from the New England Journal of Medecine, 248: 1-5, 1 January 1953,

Massachusetts Medical Society, 1953, p. 14.

69. ConnelLy, « Children are not... », p. 14. nauté au sens ethnique, culturel et religieux. Le tribunal emploie d'ailleurs un aumônier et les pratiques religieuses institutionnalisées sont reconnues comme un moyen légitime d'amélioration de soi et de sa conduite.

Du point de vue des jugements, s'il ne faut pas négliger les placements et la dure réalité de l'enfermement ${ }^{63}$, la mesure-phare, surtout à Boston, est la probation (ou remise aux parents sous liberté surveillée, pour reprendre les termes de la procédure française). Plus de $60 \%$ des mineurs jugés délinquants ou indisciplinés par le TE de Boston, en 1951, sont mis en probation ${ }^{64}$. Celle-ci est appliquée à l'anglo-saxonne de manière pragmatique, quelquefois assez interventionniste à travers des " conditions de probation " plus ou moins contraignantes. Le but est d'aboutir à un " travail constructif ${ }^{65}$ ". Deux exemples:

- En avril 1948, un garçon de 13 ans tire, à plusieurs reprises, sur un écran de cinéma avec un fusil à air comprimé; il est mis en probation pour neuf mois et doit, entre autres obligations, visiter un hôpital pour constater les blessures que peut infliger ce type d'armes ${ }^{66}$.

- En 1950, à la première nuit d'été, une « fille plutôt jolie » de 16 ans est arrêtée, à minuit et demi, dans le jardin public de Boston, pour "fornication » avec un garde-côte de 19 ans; elle est mise en probation pour un an sous la condition de ne plus se rendre à Boston et de ne plus fréquenter les militaires ${ }^{67}$.

\section{Conclusion}

Le sceau de la BJC (ill. 11) représente une justice qui s'abreuve à la miséricorde et s'enrichit de la clémence: «La miséricorde ne se commande pas. Elle tombe comme la douce pluie du ciel. " La citation choisie comme devise est tirée du Marchand de Venise de Shakespeare, de la scène où Portia s'adresse à Shylock le Juif, venu réclamer en justice son dû, une livre de chair de son débiteur. Portia défend la clémence face à la stricte application du droit qu'il réclame. Au Dieu de la Loi doit succéder le Dieu d'amour du Nouveau Testament.

Lors de la célébration des 45 ans de la BJC, en 1951, le rôle du tribunal est ainsi illustré par les paroles de Jésus : " [Laissez venir à moi les petits enfants et] ne les en empêchez pas, car le royaume de Dieu est pour ceux qui leur ressemblent ${ }^{68}$. » Accueillir les plus faibles, les accompagner et " alléger leur fardeau $^{69}$ " sur le chemin de la vie, voici le programme d'avenir et d'espoir des juvenile courts. 


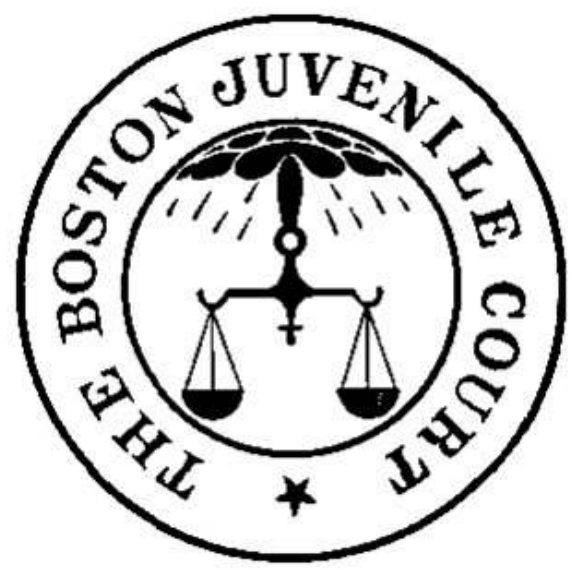

Illustration 11. Sceau et devise de la Boston Juvenile Court: « The quality of mercy is not strain'd, it droppeth as the gentle rain from heaven » - " La miséricorde ne se commande pas, Elle tombe comme la douce pluie du ciel ». Source: The Citizenship Training Group, Inc., Research Department (compiler and ed.), Boston Juvenile Court: Annual Report, 1951, page de titre.

En France, laïcité oblige, la référence néotestamentaire est nettement moins explicite, bien que... Dans le bestseller de 1954 de Gilbert Cesbron (futur secrétaire général du Secours catholique français), dont est tiré le film Chiens perdus sans collier, le juge Lamy regarde, par la fenêtre, la Sainte Chapelle surplombant le palais de justice de Paris où il exerce, s'abandonnant au flot du lyrisme, à la pensée de la couronne d'épines du Christ que la chapelle abrite: "C'est un otage: elle est là pour témoigner, pour crier qu'il n'y a pas de Justice sans Amour $^{70}$ !" Dans le palais de justice, une tapisserie (ill. 12) orne réellement, à partir de 1946, la salle d'audience du TE de la Seine. Au centre domine une figure christique, tenant un glaive en forme de croix, séparant les bons des méchants: "volonté plus ou moins consciente ", après-guerre, de "réintroduire le Christ dans les prétoires ${ }^{71}$ ?"

À travers le thème choisi du jugement dernier, il apparaît que les TE français gardent un caractère plus formel, plus juridique, et ne vont pas aussi loin que leurs homologues américains, saturés de valeurs humanistes, dans l'abandon du pénal ${ }^{72}$. S'ils insistent sur la compréhension du mineur plus que sur sa culpabilité, la réhabilitation et non la peine rétributive, ils séparent néanmoins toujours les bons des méchants.

70. Cesbron Gilbert, Chiens perdus sans collier, Paris, Robert Laffont, 1954, p. 293.

71. BOURQuIN Jacques, "L'image du jugement dernier dans la salle d'audience du tribunal pour enfants de Paris: une tapisserie de Georges Devêche ", RHEI, n 4 , 2002, p. 175.

72. Dans les discours en tous cas, les pratiques étant plus nuancées et moins radicales que le " mythe " construit par les child savers (qui se retournera contre eux à travers les accusations de laxisme), comme l'a montré Tanenhaus David S., Juvenile Justice... 


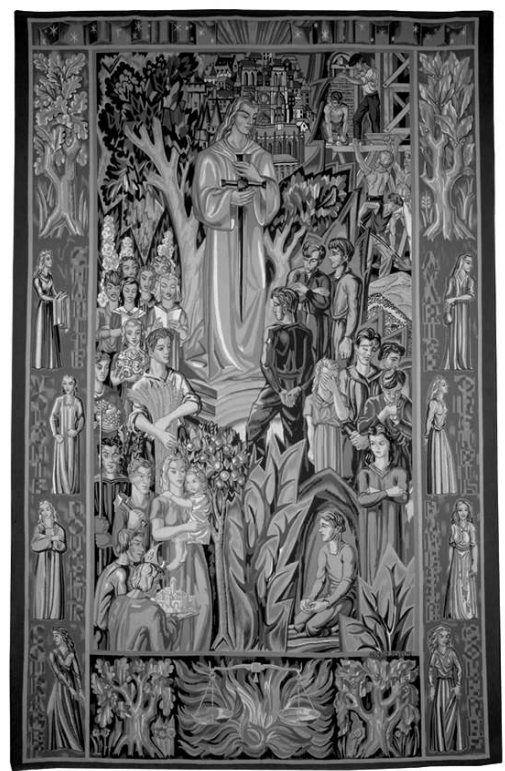

Illustration 12. Georges Devêche, Tapisserie pour la salle d'audience du tribunal pour enfants de la Seine, commandée au début de 1943 et achevée en 1946. Photographie: Michel Basdevant. Source: Bourquin Jacques, "L'image du jugement dernier dans la salle d'audience du tribunal pour enfants de Paris: une tapisserie de Georges Devêche ", Revue d'histoire de l'enfance « irrégulière », $n^{\circ} 4,2002$, p. 169.

À propos des TE, il a été question d'amour paternel, incarné par le juge, d'amour maternel, surtout aux États-Unis influencés par le courant maternaliste, d'amour fraternel enfin, avec l'agent de probation (ou encore les associations de Big Brothers et Big Sisters, Grands Frères et Grandes Sours). Mais cet amour familial, parfois qualifié de bourgeois dans ses modes d'imposition, est subsumé sous l'amour du prochain, qui, modernisé, démocratisé et plus ou moins déchristianisé, joue un rôle fédérateur, par-delà les questions de classes, en posant les idéaux de compréhension mutuelle et d'échange entre sujets.

Le succès mondial du TE est rendu possible, entre autres, par cette idéologie, qui justifie et assure la bonne marche des nouveaux dispositifs, comme la probation et ses variantes. Ces dispositifs plus souples, plus modulables, plus insidieux peut-être, répondent aux besoins et rentrent dans la logique de sociétés démocratiques de moins en moins disciplinaires au sens de Foucault, où la 
prison, si elle continue d'être utilisée, sert de moins en moins de modèle global et d'horizon théorique ${ }^{73}$.

Le comparatisme rend compte de la nature profonde du TE. À la fois appareil répressif et idéologique d'État ${ }^{74}$, la justice des mineurs fonctionne de moins en moins à la violence et de plus en plus à l'idéologie, la dose d'idéologie variant selon les pays. Elle catalyse ainsi l'évolution judiciaire et rend visibles certaines mutations du monde occidental et de ses manières de penser et de contrôler.

73. L'État carcéral américain actuel, intégrant les mineurs, et dont nous parlions en introduction, semblerait contredire cette interprétation. Sans dépasser le cadre de cet article, rappelons simplement que les innovations pénales se font aujourd'hui largement en dehors de la prison, qui, du coup, a pu être qualifiée de post-disciplinaire, puisque ramenée à la « gestion des déchets " (waste management), perdant l'idéal, ou l'illusion, de la réhabilitation. L'incarcération de masse ne doit pas non plus cacher le poids, statistiquement encore plus lourd, des autres formes de pénalités probation et ses variantes, du milieu ouvert au bracelet électronique, etc.

74. Voir Althusser Louis, « Idéologie et appareils idéologiques d'État (Notes pour une recherche) ", avril 1970, Positions, Paris, Éditions sociales, 1982, p. 79-137. 